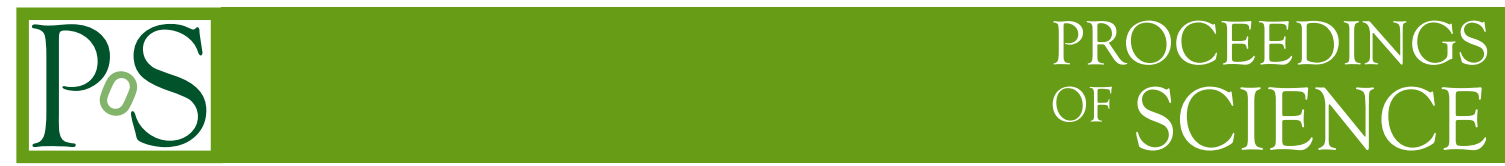

\title{
Kaon Physics: Theory Overview
}

\author{
Antonio Pich* \\ IFIC, Universitat de València-CSIC, Apt. Correus 22085, E-46071 València, Spain \& \\ Physik Dep. and Inst. for Advanced Study, Tech. Univ. München, D-85748 Garching, Germany \\ E-mail: Antonio.Picheific.uv.es
}

\begin{abstract}
Kaon decays have played a key role in the construction of the Standard Model and continue to be an important testing ground of the electroweak flavour theory. They can provide new signals of CP-violation phenomena and, perhaps, a window into physics beyond the Standard Model. The interplay of long-distance QCD effects in strangeness-changing transitions can be analyzed, combining the short-distance Operator Product Expansion with Chiral Perturbation Theory techniques. A brief overview is presented, focusing on a few selected decay modes. A more detailed and comprehensive review can be found in Ref. [1].
\end{abstract}

The XIth International Conference on Heavy Quarks and Leptons,

June 11-15, 2012

Prague, Czech Republic

${ }^{*}$ Speaker. 


\section{Theoretical Framework}

Kaons have been at the center of many fundamental developments in particle physics, playing a key role in the construction of what we now call the Standard Model (SM): the introduction of internal flavour quantum numbers (strangeness) [2, 3], parity violation [4, 5], meson-antimeson mixing [6, 7], quark mixing [8,9], the discovery of $\mathrm{CP}$ violation [10], and the suppression of flavour-changing neutral currents and the GIM mechanism [11]. High-precision experiments on rare kaon decays provide sensitivity to short-distance scales $\left(c, t, W^{ \pm}, Z\right)$ and offer the exciting possibility of unravelling new physics beyond the SM. Searching for forbidden flavour-changing processes beyond the $10^{-10}$ level $\left[\operatorname{Br}\left(K_{L} \rightarrow e^{ \pm} \mu^{\mp}\right)<4.7 \times 10^{-12}[12], \operatorname{Br}\left(K_{L} \rightarrow e^{ \pm} e^{ \pm} \mu^{\mp} \mu^{\mp}\right)<\right.$ $4.12 \times 10^{-11}$ [13], $\operatorname{Br}\left(K^{+} \rightarrow \pi^{+} \mu^{+} e^{-}\right)<1.3 \times 10^{-11}$ [14], $\operatorname{Br}\left(K^{+} \rightarrow \pi^{+} \mu^{-} e^{+}\right)<5.2 \times 10^{-10}$ [15] (90\% C.L.)], one is actually exploring energy scales above the $10 \mathrm{TeV}$ region. The study of allowed (but highly suppressed) decay modes provides, at the same time, very interesting tests of the SM itself. Electromagnetic-induced non-leptonic weak transitions and higher-order weak processes are a useful tool to improve our understanding of the interplay among electromagnetic, weak and strong interactions. In addition, new signals of $\mathrm{CP}$ violation, which would help to elucidate the source of CP-violating phenomena, can be looked for.

The theoretical analysis of kaon decays is highly non-trivial. While the underlying flavourchanging transitions among the constituent quarks are associated with the electroweak scale, the corresponding hadronic amplitudes are governed by the long-distance behaviour of the strong interactions, i.e., the confinement regime of QCD. The short-distance approach to weak transitions makes use of the asymptotic freedom property of QCD to successively integrate out the fields with heavy masses down to scales $\mu<m_{c}$. Using the operator product expansion (OPE) and renormalization-group techniques, one gets an effective $\Delta S=1$ Hamiltonian [16]

$$
\mathscr{H}_{\mathrm{eff}}^{\Delta S=1}=\frac{G_{F}}{\sqrt{2}} V_{u d} V_{u s}^{*} \sum_{i} C_{i}(\mu) Q_{i}+\text { h.c., } \quad C_{i}(\mu)=z_{i}(\mu)-y_{i}(\mu) \frac{V_{t d} V_{t s}^{*}}{V_{u d} V_{u s}^{*}},
$$

which is a sum of local four-fermion operators $Q_{i}$, constructed with the light degrees of freedom $\left(u, d, s ; e, \mu, v_{l}\right)$, modulated by Wilson coefficients $C_{i}(\mu)$ which are functions of the heavy $(Z, W, t, b, c, \tau)$ masses. The CP-violating decay amplitudes are proportional to the components $y_{i}(\mu)$. The overall renormalization scale $\mu$ separates the short- $(M>\mu)$ and long-distance $(m<\mu)$ contributions, which are contained in $C_{i}(\mu)$ and $Q_{i}$, respectively. The Wilson coefficients are fully known at the next-to-leading order (NLO) [17, 18]; this includes all corrections of $\mathscr{O}\left(\alpha_{s}^{n} t^{n}\right)$ and $\mathscr{O}\left(\alpha_{s}^{n+1} t^{n}\right)$, where $t \equiv \log \left(M_{1} / M_{2}\right)$ refers to the logarithm of any ratio of heavy mass scales $\left(M_{1,2} \geq \mu\right)$. In order to calculate the kaon decay amplitudes, we also need to know the nonperturbative matrix elements of the operators $Q_{i}$ between the initial and final states.

The low-energy strong interactions are better understood with symmetry considerations, because the lightest pseudoscalar mesons correspond to the octet of Goldstone bosons associated with the dynamical chiral symmetry breaking of QCD: $S U(3)_{L} \otimes S U(3)_{R} \rightarrow S U(3)_{V}$. Their dynamical properties can then be worked out systematically through an effective Lagrangian. The quark and gluon fields of QCD are replaced by a unitary matrix $U(\phi) \equiv \exp (i \sqrt{2} \Phi / F)$, parameterizing the Goldstone excitacions over the vacuum quark condensate $\left\langle\bar{q}_{L}^{j} q_{R}^{i}\right\rangle(i, j=u, d, s)$. The Chiral Perturbation Theory $[19,20,21,22](\chi \mathrm{PT})$ formulation of the Standard Model is given by the most 


\begin{tabular}{ccc} 
Energy & Fields & Effective Theory \\
\hline \multirow{3}{*}{$\begin{array}{c}\begin{array}{c}W, Z, \gamma, g \\
\tau, \mu, e, v_{i} \\
t, b, c, s, d, u\end{array} \\
\downarrow\end{array}$} & Standard Model \\
$\lesssim m_{c}$ & $\begin{array}{c}\gamma, g ; \mu, e, v_{i} \\
s, d, u\end{array}$ \\
$\downarrow$ & $\mathscr{L}_{\mathrm{QCD}}^{n_{f}=3}, \mathscr{H}_{\mathrm{eff}}^{\Delta S=1,2}$ \\
$m_{K}$ & $\begin{array}{c}\gamma ; \mu, e, v_{i} \\
\pi, K, \eta\end{array}$ & $\chi \mathrm{PT}$
\end{tabular}

Figure 1: Evolution from $M_{W}$ to the kaon mass scale.
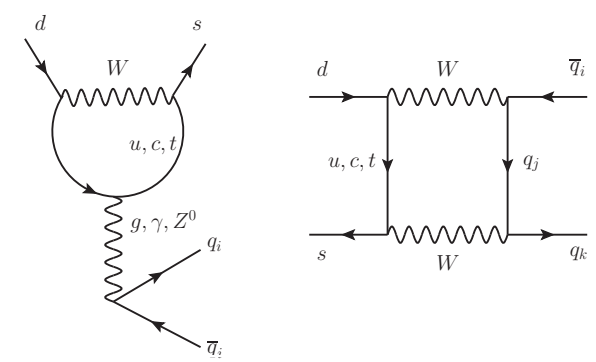

Figure 2: Short-distance Feynman diagrams.

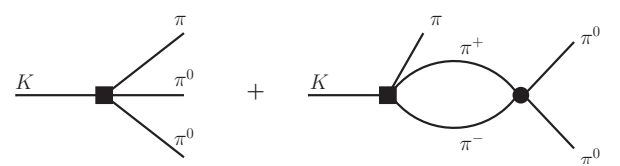

Figure 3: Long-distance Feynman diagrams.

general effective Lagrangian, involving the matrix $U(\phi)$, which is consistent with chiral symmetry. The Lagrangian can be organized in terms of increasing powers of momenta (derivatives) and quark masses over the chiral symmetry-breaking scale $\left(\Lambda_{\chi} \sim 1 \mathrm{GeV}\right)$. All short-distance information is encoded in the low-energy couplings (LECs) of the $\chi \mathrm{PT}$ operators. At lowest order (LO), $\mathscr{O}\left(p^{2}\right)$, the strong interactions are fully parameterized in terms of only two LECs: the pion decay constant $F \simeq F_{\pi}=92.4 \mathrm{MeV}$ and $B_{0} \simeq-<\bar{u} u>/ F^{2}$, which accounts for the explicit chiral symmetry breaking through the quark masses. Ten additional LECs $L_{i}$ are needed at $\mathscr{O}\left(p^{4}\right)$.

Non-leptonic weak interactions with $\Delta S=1$ are incorporated as a perturbation to the strong $\chi$ PT Lagrangian. At LO the most general effective Lagrangian, with the same $S U(3)_{L} \otimes S U(3)_{R}$ transformation properties as the short-distance Hamiltonian (1.1), contains three terms [1]:

$$
\mathscr{L}_{2}^{\Delta S=1}=-\frac{G_{F}}{\sqrt{2}} V_{u d} V_{u s}^{*}\left\{g_{8}\left\langle\lambda L_{\mu} L^{\mu}\right\rangle+g_{27}\left(L_{\mu 23} L_{11}^{\mu}+\frac{2}{3} L_{\mu 21} L_{13}^{\mu}\right)+e^{2} g_{8} g_{\mathrm{ew}} F^{6}\left\langle\lambda U^{\dagger} Q U\right\rangle+\text { h.c. }\right\},
$$

where $L_{\mu}=i F^{2} U^{\dagger} D_{\mu} U$ represents the octet of $V-A$ currents, $\lambda \equiv\left(\lambda_{6}-i \lambda_{7}\right) / 2$ projects onto the $\bar{s} \rightarrow \bar{d}$ transition, $Q=\frac{1}{3} \operatorname{diag}(2,-1,-1)$ is the quark charge matrix and $\langle\cdots\rangle$ denotes the 3dimensional flavour trace. The LECs $g_{8}$ and $g_{27}$ measure the strength of the two parts of $\mathscr{H}_{\text {eff }}^{\Delta S=1}$ transforming as $\left(8_{L}, 1_{R}\right)$ and $\left(27_{L}, 1_{R}\right)$, respectively, under chiral rotations, while $g_{\text {ew }}$ accounts for the electromagnetic penguin operators.

The $\chi \mathrm{PT}$ framework determines the most general form of the $\mathrm{K}$ decay amplitudes, compatible with chiral symmetry, in terms of the LECs multiplying the relevant chiral operators. These LECs, which encode the short-distance dynamics, can be determined phenomenologically and/or calculated in the limit of a large number of QCD colours (matching). Chiral loops generate nonpolynomial contributions, with logarithms and threshold factors as required by unitarity. Fig. 1 shows schematically the procedure used to evolve down from $M_{W}$ to $m_{K}$. While the OPE resums the short-distance logarithmic corrections $\log (M / \mu)$, the $\chi$ PT loops take care of the large infrared $\operatorname{logarithms~} \log \left(\mu / m_{\pi}\right)$ associated with unitarity corrections (final-state interactions). 


\begin{tabular}{cc}
\hline \hline$\left|g_{\mu} / g_{e}\right|$ & Source \\
\hline $1.0021 \pm 0.0016$ & $\pi \rightarrow \mu / e$ \\
$0.9978 \pm 0.0018$ & $K \rightarrow \mu / e$ \\
$1.0010 \pm 0.0025$ & $K \rightarrow \pi \mu / e$ \\
$1.0018 \pm 0.0014$ & $\tau \rightarrow \mu / e$ \\
\hline \hline
\end{tabular}

Table 1: Determinations of the ratio of the $\mu^{-}$ and $e^{-}$couplings to the $W$.

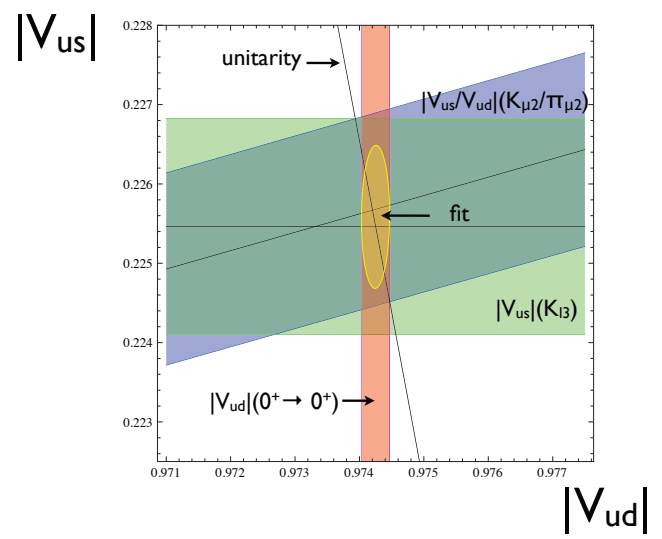

Figure 4: Constraints on $\left|V_{u d}\right|$ and $\left|V_{u s}\right|$ from $K_{\ell 3}$ decays (horizontal), $0^{+} \rightarrow 0^{+}$nuclear decays (vertical) and $K_{\mu 2} / \pi_{\mu 2}$ (oblique band) [33].

\section{Leptonic and Semileptonic Decays.}

Leptonic and semileptonic kaon decays are well understood theoretically, including electromagnetic corrections. Strong interactions only appear through the hadronic matrix elements of the left-handed weak current, which can be precisely studied within $\chi \mathrm{PT}$ and with lattice simulations.

The ratios $R_{e / \mu}^{(P)} \equiv \Gamma\left[P^{-} \rightarrow e^{-} \bar{v}_{e}(\gamma)\right] / \Gamma\left[P^{-} \rightarrow \mu^{-} \bar{v}_{\mu}(\gamma)\right] \quad(P=\pi, K)$ have been calculated $[23,24]$ and measured $[25,26,27,28,29]$ with high accuracy, allowing for a test of chargedcurrent lepton universality at the $0.2 \%$ level. As shown in Table 1, similar precisions have been achieved in $K \rightarrow \pi \ell v_{\ell}$ [30] and $\tau \rightarrow v_{\tau} \ell v_{\ell}$ [31] decays.

The ratio of radiative inclusive decay rates $\Gamma\left[K^{-} \rightarrow \mu^{-} \bar{v}_{\mu}(\gamma)\right] / \Gamma\left[\pi^{-} \rightarrow \mu^{-} \bar{v}_{\mu}(\gamma)\right]$ provides information on the quark mixing matrix [30,32]. With a careful treatment ot electromagnetic and isospin-violating corrections, one extracts $\left|V_{u s} / V_{u d}\right|\left|F_{K} / F_{\pi}\right|=0.2763 \pm 0.0005$ [33]. Taking for the ratio of meson decay constants the lattice average $F_{K} / F_{\pi}=1.193 \pm 0.006$ [34], this gives

$$
\left|V_{u s} / V_{u d}\right|=0.2316 \pm 0.0012 .
$$

The most recent $K_{\ell 3}$ experiments have resulted in improved precision and significant shifts in the branching fractions [36]. Including electromagnetic and isospin-breaking corrections [37, 38], one obtains $\left|V_{u s} f_{+}(0)\right|=0.2163 \pm 0.0005$ [30], with $f_{+}(0)=1+\mathscr{O}\left[\left(m_{s}-m_{u}\right)^{2}\right]$ the $K^{0} \rightarrow \pi^{-} \ell^{+} v_{\ell}$ vector form factor. The exact value of $f_{+}(0)$ has been thoroughly investigated since the first precise estimate by Leutwyler and Roos, $f_{+}(0)=0.961 \pm 0.008$ [39]. While analytical calculations based on $\chi$ PT obtain higher values [40, 41], owing to the large ( $\sim 0.01)$ 2-loop corrections [42], lattice results [34] tend to agree with the Leutwyler-Roos estimate. Taking as reference value the most recent and precise lattice result [43], $f_{+}(0)=0.960 \pm 0.006$, one obtains [1]

$$
\left|V_{u s}\right|=0.2255 \pm 0.0005_{\exp } \pm 0.0012_{\text {th }} .
$$

Together with $\left|V_{u d}\right|=0.97425 \pm 0.00022$ [35] and the negligible $\left|V_{u b}\right|$ contribution [36], the determinations (2.1) and (2.2) imply an stringent test of the unitarity of the quark mixing matrix [33]:

$$
\Delta_{\mathrm{CKM}}=\left|V_{u d}\right|^{2}+\left|V_{u s}\right|^{2}+\left|V_{u b}\right|^{2}-1=0.0001 \pm 0.0006 .
$$




\section{Nonleptonic Decays and Direct CP Violation: $\varepsilon^{\prime} / \varepsilon$}

The measured $A(K \rightarrow \pi \pi)_{I}$ decay amplitudes show a strong enhancement of the octet $\Delta I=\frac{1}{2}$ transition amplitude into a $2 \pi$ final state with isospin $I=0:\left|A_{0} / A_{2}\right| \approx 22$. In the $\chi$ PT framework this manifests as a huge difference between the LECs in Eq. (1.2). A LO fit to the data gives $\left|g_{8}\right| \simeq 5.0$ and $\left|g_{27}\right| \simeq 0.285$. Part of the enhancement originates in the strong rescattering of the final pions, which at one loop increases $A_{0}$ by roughly $35 \%$; taking the $\chi$ PT 1-loop contributions into account, one finds a sizeably smaller octet coupling (the central values change slightly to 3.61 and 0.297 , respectively, if isospin violation is included) [1, 44, 45]:

$$
\left|g_{8}\right|=3.62 \pm 0.28, \quad\left|g_{27}\right|=0.286 \pm 0.28 .
$$

In the absence of QCD corrections, the SM ( $W$ exchange) implies $g_{8}=g_{27}=\frac{3}{5}$, very far from the phenomenologically required values. The computed short-distance QCD corrections show the needed qualitative trend to understand the data, but a proper calculation of the hadronic matrix elements of the relevant $Q_{i}$ operators is still lacking. The matching of the effective descriptions $\mathscr{H}_{\text {eff }}^{\Delta S=1}$ (short-distance) and $\mathscr{L}_{2}^{\Delta S=1}(\chi \mathrm{PT})$ can be done in the large- $N_{C}$ limit with the result $g_{8}^{\infty}=$ $1.13 \pm 0.18$ and $g_{27}^{\infty}=0.46 \pm 0.01$ [1], which shows the relevance of the missing NLO corrections in $1 / N_{C}$. Lattice simulations have recently achieved a quite successful description of $A_{2}\left(g_{27}\right)$ [46], but a real quantitative understanding of $A_{0}\left(g_{8}\right)$ remains still problematic [47].

The situation is much better fot the ratio $[48,49,50,51]\left[\eta_{a b} \equiv A\left(K_{L} \rightarrow \pi^{a} \pi^{b}\right) / A\left(K_{S} \rightarrow \pi^{a} \pi^{b}\right)\right]$

$$
\operatorname{Re}\left(\varepsilon^{\prime} / \varepsilon\right)=\frac{1}{3}\left(1-\left|\frac{\eta_{00}}{\eta_{+-}}\right|\right)=(16.8 \pm 2.0) \times 10^{-4},
$$

which demonstrates the existence of direct CP violation in the $K \rightarrow 2 \pi$ decay amplitudes. When $\mathrm{CP}$ violation is turned on, the amplitudes $A_{I}$ acquire imaginary parts. To first order in CP violation,

$$
\varepsilon^{\prime}=-\frac{i}{\sqrt{2}} e^{i\left(\chi_{2}-\chi_{0}\right)} \frac{\operatorname{Re} A_{2}}{\operatorname{Re} A_{0}}\left[\frac{\operatorname{Im} A_{0}}{\operatorname{Re} A_{0}}-\frac{\operatorname{Im} A_{2}}{\operatorname{Re} A_{2}}\right],
$$

where the strong phases $\chi_{I}$ can be identified with the S-wave $\pi \pi$ scattering phase shifts at $\sqrt{s}=m_{K}$, up to isospin-breaking effects $[44,45]$. The phase $\phi_{\varepsilon^{\prime}}=\chi_{2}-\chi_{0}+\pi / 2=(42.5 \pm 0.9)^{\circ}$ is very close to the so-called superweak phase, $\phi_{\varepsilon} \approx \tan ^{-1}\left[2\left(m_{K_{L}}-m_{K_{S}}\right) /\left(\Gamma_{K_{S}}-\Gamma_{K_{L}}\right)\right]=(43.51 \pm 0.05)^{\circ}$, implying that $\cos \left(\phi_{\varepsilon^{\prime}}-\phi_{\varepsilon}\right) \approx 1$. The CP-conserving amplitudes $\operatorname{Re} A_{I}$ can be set to their experimentally determined values, avoiding in this way the large uncertainties associated with the hadronic matrix elements of the four-quark operators in $\mathscr{H}_{\text {eff }}^{\Delta S=1}$. Thus, one only needs a first-principle calculation of the CP-odd amplitudes $\operatorname{Im} A_{0}$ and $\operatorname{Im} A_{2}$; the first one is completely dominated by the strong penguin operator $Q_{6}$, while the leading contribution to the second one comes from the electromagnetic penguin $Q_{8}$. Fortunately, those are precisely the only operators that are well approximated through a large- $N_{C}$ estimate of LECs, because their anomalous dimensions are leading in $1 / N_{C}$. Owing to the large ratio $\operatorname{Re} A_{0} / \operatorname{Re} A_{2}$, isospin violation plays also an important role in $\varepsilon^{\prime} / \varepsilon$ [45]. The one-loop $\chi \mathrm{PT}$ enhancement of the isoscalar amplitude [52, 53] destroys an accidental LO cancellation of the two terms in (3.3) [54, 55, 56], bringing the SM prediction of $\varepsilon^{\prime} / \varepsilon$ in good agreement with the experimental measurement in Eq. (3.2) [52, 53, 57]:

$$
\operatorname{Re}\left(\varepsilon^{\prime} / \varepsilon\right)=\left(19 \pm 2_{\mu}^{+9}+6_{m_{s}} \pm 6_{1 / N_{C}}\right) \times 10^{-4} .
$$



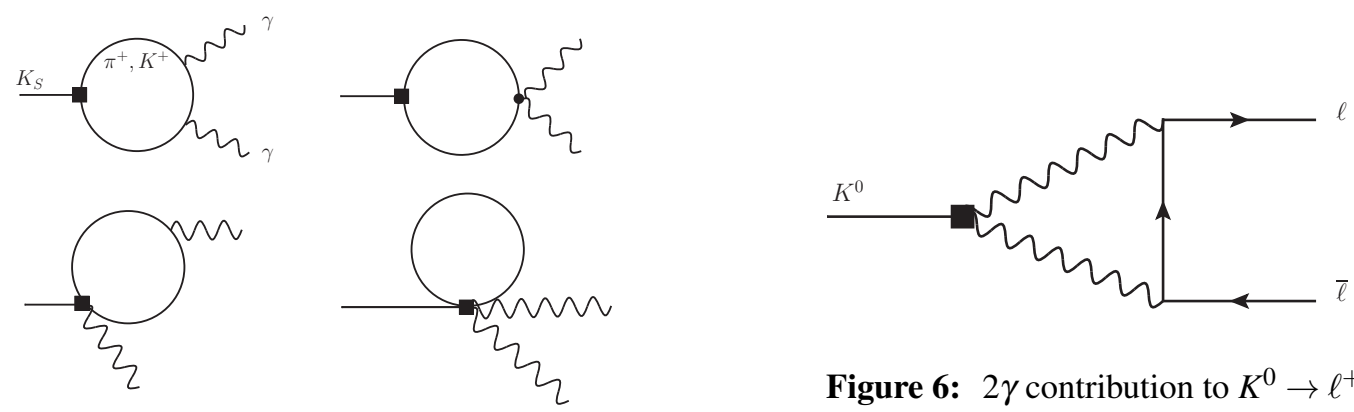

Figure 6: $2 \gamma$ contribution to $K^{0} \rightarrow \ell^{+} \ell^{-}$.

Figure 5: Lowest-order contributions to $K_{S} \rightarrow \gamma \gamma$.

\section{Rare and Radiative Decays}

Kaon decays mediated by flavour-changing neutral currents are suppressed in the SM and their main interest, other than their own understanding, relies on the possible observation of new physics effects. Most of these processes are dominated by long-distance contributions; however, there are also processes governed by short-distance amplitudes, such as $K \rightarrow \pi \nu \bar{v}$.

\section{1 $K^{0} \rightarrow \gamma \gamma$ and $K^{0} \rightarrow \ell^{+} \ell^{-}$}

The symmetry constraints do not allow any tree-level $K_{1}^{0} \gamma \gamma$ coupling at $\mathscr{O}\left(p^{4}\right)\left(K_{1,2}^{0}\right.$ are the CPeven and CP-odd states). The decay $K_{S} \rightarrow \gamma \gamma$ proceeds then through a one-loop amplitude, with intermediate $\pi^{+} \pi^{-}$, which is necessarily finite because there are no counterterms to renormalize divergences. The resulting $\mathscr{O}\left(p^{4}\right)$ prediction [58, 59], $\operatorname{Br}\left(K_{S} \rightarrow \gamma \gamma\right)=2.0 \times 10^{-6}$, is slightly lower than the experimental measurement $\operatorname{Br}\left(K_{S} \rightarrow \gamma \gamma\right)=(2.63 \pm 0.17) \times 10^{-6}$ [36]. Full agreement is obtained at $\mathscr{O}\left(p^{6}\right)$, once rescattering corrections $\left(K_{S} \rightarrow \pi \pi \rightarrow \pi^{+} \pi^{-} \rightarrow \gamma \gamma\right)$ are included [60].

The 2-loop amplitude $K_{S} \rightarrow \gamma^{*} \gamma^{*} \rightarrow \ell^{+} \ell^{-}$is also finite [61] because chiral symmetry forbids any $\mathrm{CP}$-invariant local contribution at this order. The predicted rates, $\operatorname{Br}\left(K_{S} \rightarrow e^{+} e^{-}\right)=2.1 \times 10^{-14}$ and $\operatorname{Br}\left(K_{S} \rightarrow \mu^{+} \mu^{-}\right)=5.1 \times 10^{-12}$ [61], are well below the experimental upper bounds $\operatorname{Br}\left(K_{S} \rightarrow\right.$ $\left.e^{+} e^{-}\right)<9 \times 10^{-9}$ and $\operatorname{Br}\left(K_{S} \rightarrow \mu^{+} \mu^{-}\right)<3.2 \times 10^{-7}$ (90\% C.L.) [36]. This calculation allows us to compute the longitudinal polarization $P_{L}$ of either muon in the decay $K_{L} \rightarrow \mu^{+} \mu^{-}$, a CPviolating observable which in the $\mathrm{SM}$ is dominated by indirect CP violation from $K^{0}-\bar{K}^{0}$ mixing. One finds $\left|P_{L}\right|=(2.6 \pm 0.4) \times 10^{-3}[61]$.

\section{$4.2 K \rightarrow \pi \gamma \gamma$}

Again, the symmetry constraints do not allow any tree-level contribution to $K_{2} \rightarrow \pi^{0} \gamma \gamma$ from $\mathscr{O}\left(p^{4}\right)$ terms in the Lagrangian. The decay amplitude is therefore determined by a finite loop calculation $[62,63,64]$. Due to the large absorptive $\pi^{+} \pi^{-}$contribution, the spectrum in the invariant mass of the two photons is predicted to have a very characteristic behaviour (dotted line in Fig. 7), peaked at high values of $m_{\gamma \gamma}$. The agreement with the measured distribution [65] is remarkably good. However, the $\mathscr{O}\left(p^{4}\right)$ prediction for the rate, $\mathrm{BR}\left(K_{L} \rightarrow \pi^{0} \gamma \gamma\right)=6.8 \times 10^{-7}$ [62], is significantly smaller than the present PDG average [36]

$$
\mathrm{BR}\left(K_{L} \rightarrow \pi^{0} \gamma \gamma\right)=(1.27 \pm 0.03) \times 10^{-6},
$$




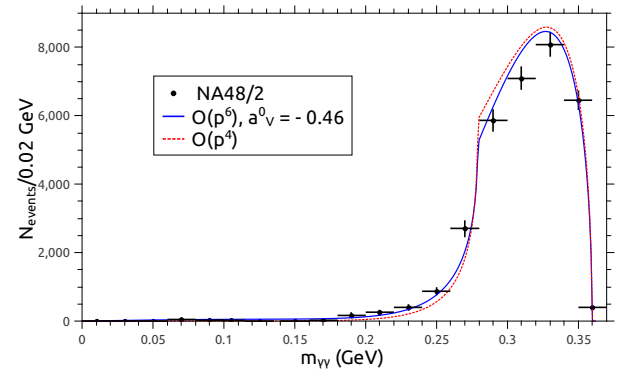

Figure 7: $K_{L} \rightarrow \pi^{0} \gamma \gamma$ spectra at $\mathscr{O}\left(p^{4}\right)$ and $\mathscr{O}\left(p^{6}\right)$ in $\chi$ PT. The data are from Ref. [65].

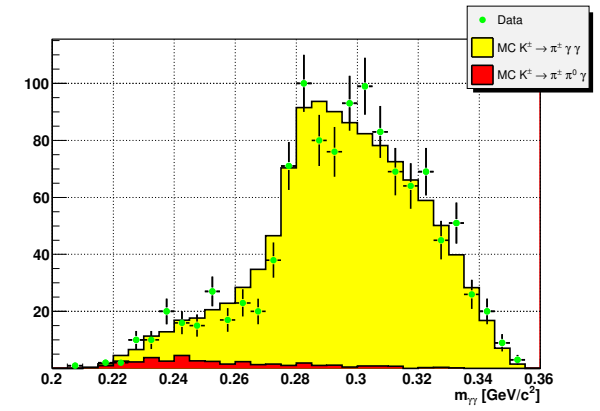

Figure 8: Measured $K^{ \pm} \rightarrow \pi^{ \pm} \gamma \gamma$ spectrum [71].

indicating that higher-order corrections are sizeable. Unitarity corrections from $K_{L} \rightarrow \pi^{+} \pi^{-} \pi^{0}$ $[66,67]$ and local vector-exchange contributions $[66,68]$ restore the agreement at $\mathscr{O}\left(p^{6}\right)$.

A quite similar spectrum is predicted [69] for the charged mode $K^{ \pm} \rightarrow \pi^{ \pm} \gamma \gamma$, but in this case there is a free LEC already at $\mathscr{O}\left(p^{4}\right) \cdot \mathscr{O}\left(p^{6}\right)$ corrections have been also investigated [70]. Both the spectrum and the rate can be correctly reproduced $[71,72]$.

\section{3 $K_{L} \rightarrow \pi^{0} e^{+} e^{-}$}

This decay is an interesting process in looking for new CP-violating signatures, because $K_{2}^{0} \rightarrow$ $\pi^{0} \gamma^{*}$ violates CP $[69,73]$. The CP-conserving amplitude proceeds through a $2 \gamma$ intermediate state and is suppressed by an additional power of $\alpha$. Using the $K_{L} \rightarrow \pi^{0} \gamma \gamma$ data, the CP-conserving rate is found to be below $10^{-12}$ [1]. The $K_{L} \rightarrow \pi^{0} e^{+} e^{-}$transition is then dominated by the $\mathscr{O}(\alpha)$ CP-violating contributions [69], both from $K^{0}-\bar{K}^{0}$ mixing and direct CP violation. The estimated rate $\operatorname{Br}\left(K_{L} \rightarrow \pi^{0} e^{+} e^{-}\right)=(3.1 \pm 0.9) \times 10^{-11}[1,74,75]$ is only a factor 10 smaller than the present ( $90 \%$ C.L.) upper bound of $2.8 \times 10^{-10}[76]$ and should be reachable in the near future.

\section{4 $K \rightarrow \pi v \bar{v}$}

Long-distance effects play a negligible role in $K^{+} \rightarrow \pi^{+} v \bar{v}$ and $K_{L} \rightarrow \pi^{0} v \bar{v}$. These processes are dominated by short-distance loops ( $Z$ penguin, $W$ box) involving the heavy top quark; the charged mode receives also sizeable contributions from internal charm-quark exchanges. The decay amplitudes are proportional to the hadronic matrix element of the $\Delta S=1$ vector current, which (assuming isospin symmetry) can be obtained from $K_{\ell 3}$ decays:

$$
T(K \rightarrow \pi \nu \bar{v}) \sim \sum_{i=c, t} F\left(V_{i d} V_{i s}^{*} ; x_{i}\right)\left(\bar{v}_{L} \gamma_{\mu} v_{L}\right)\left\langle\pi\left|\bar{s}_{L} \gamma^{\mu} d_{L}\right| K\right\rangle, \quad x_{i} \equiv m_{i}^{2} / M_{W}^{2} .
$$

The small long-distance and isospin-violating corrections can be estimated within $\chi \mathrm{PT}$. The $K_{L} \rightarrow$ $\pi^{0} v \bar{v}$ transition violates $\mathrm{CP}$ and is completely dominated by direct $\mathrm{CP}$ violation, the contribution from $K^{0}-\bar{K}^{0}$ mixing being only of the order of $1 \%$. Taking the CKM inputs from global fits, one predicts $\operatorname{Br}\left(K_{L} \rightarrow \pi^{0} v \bar{v}\right)=(2.4 \pm 0.4) \times 10^{-11}$ and $\operatorname{Br}\left(K^{+} \rightarrow \pi^{+} v \bar{v}\right)=(0.78 \pm 0.08) \times 10^{-10}$ [77, 78]. The uncertainties are largely parametrical, due to CKM input, $m_{c}, m_{t}$ and $\alpha_{s}\left(M_{Z}\right)$.

The $K^{+}$mode has been observed [79], while only an upper bound exists on the $K_{L}$ one [80]:

$$
\left.\operatorname{Br}\left(K^{+} \rightarrow \pi^{+} v \bar{v}\right)=\left(1.73_{-1.05}^{+1.15}\right) \times 10^{-10}, \quad \operatorname{Br}\left(K_{L} \rightarrow \pi^{0} v \bar{v}\right)<2.6 \times 10^{-8} \text { (90\% C.L. }\right) .
$$


New experiments, aiming to reach $\mathscr{O}(100)$ events (assuming SM rates), are under development at CERN (NA62) and J-PARC (K0TO) for charged and neutral modes, respectively. Increased sensitivities could be obtained through the recent ORKA proposal for a $K^{+} \rightarrow \pi^{+} v \bar{v}$ experiment at Fermilab and the higher kaon fluxes available at Project-X [81].

\section{Acknowledgments}

Work supported in part by the Spanish Government [grants FPA2007-60323, FPA2011-23778 and CSD2007-00042 (Consolider Project CPAN)], the Generalitat Valenciana [Prometeo/2008/069] and the Alexander von Humboldt Foundation.

\section{References}

[1] V. Cirigliano, G. Ecker, H. Neufeld, A. Pich and J. Portolés, Rev. Mod. Phys. 84 (2012) 399 [arXiv:1107.6001 [hep-ph]].

[2] M. Gell-Mann, Phys. Rev. 92 (1953) 833.

[3] A. Pais, Phys. Rev. 86 (1952) 663.

[4] R. H. Dalitz, Phys. Rev. 94 (1954) 1046.

[5] T. D. Lee and C. -N. Yang, Phys. Rev. 104 (1956) 254.

[6] K. Lande et al., Phys. Rev. 103 (1956) 1901.

[7] W. F. Fry, J. Schneps and M. S. Swami, Phys. Rev. 103 (1956) 1904.

[8] N. Cabibbo, Phys. Rev. Lett. 10 (1963) 531.

[9] M. Kobayashi and T. Maskawa, Prog. Theor. Phys. 49 (1973) 652.

[10] J. H. Christenson, J. W. Cronin, V. L. Fitch and R. Turlay, Phys. Rev. Lett. 13 (1964) 138.

[11] S. L. Glashow, J. Iliopoulos and L. Maiani, Phys. Rev. D 2 (1970) 1285.

[12] D. Ambrose et al. [BNL Collaboration], Phys. Rev. Lett. 81 (1998) 5734 [hep-ex/9811038].

[13] A. Alavi-Harati et al. [KTeV Collaboration], Phys. Rev. Lett. 90 (2003) 141801 [hep-ex/0212002].

[14] A. Sher et al., Phys. Rev. D 72 (2005) 012005 [hep-ex/0502020].

[15] R. Appel et al., Phys. Rev. Lett. 85 (2000) 2877 [hep-ex/0006003].

[16] F. J. Gilman and M. B. Wise, Phys. Rev. D 20 (1979) 2392, 21 (1980) 3150.

[17] A. J. Buras et al., Nucl. Phys. B 400 (1993) 37 [hep-ph/9211304], 75 [hep-ph/9211321], 408 (1993) 209 [hep-ph/9303284]; Phys. Lett. B 389 (1996) 749 [hep-ph/9608365].

[18] M. Ciuchini et al., Z. Phys. C 68 (1995) 239 [hep-ph/9501265]; Phys. Lett. B 301 (1993) 263 [hep-ph/9212203]; Nucl. Phys. B 415 (1994) 403 [hep-ph/9304257].

[19] S. Weinberg, Physica A 96 (1979) 327.

[20] J. Gasser and H. Leutwyler, Nucl. Phys. B 250 (1985) 465, 517; Annals Phys. 158 (1984) 142.

[21] G. Ecker, Prog. Part. Nucl. Phys. 35 (1995) 1 [hep-ph/9501357].

[22] A. Pich, Rept. Prog. Phys. 58 (1995) 563 [hep-ph/9502366]. 
[23] W. J. Marciano and A. Sirlin, Phys. Rev. Lett. 71 (1993) 3629.

[24] V. Cirigliano and I. Rosell, Phys. Rev. Lett. 99 (2007) 231801 [arXiv:0707.3439 [hep-ph]]; JHEP 0710 (2007) 005 [arXiv:0707.4464 [hep-ph]].

[25] D. I. Britton et al., Phys. Rev. D 49 (1994) 28; Phys. Rev. Lett. 68 (1992) 3000.

[26] G. Czapek et al., Phys. Rev. Lett. 70 (1993) 17.

[27] C. Lazzeroni et al. [NA62 Collaboration], Phys. Lett. B 698 (2011) 105 [arXiv:1101.4805 [hep-ex]].

[28] F. Ambrosino et al. [KLOE Collaboration], Eur. Phys. J. C 64 (2009) 627 [Erratum-ibid. 65 (2010) 703] [arXiv:0907.3594 [hep-ex]].

[29] F. Bucci, these proceedings.

[30] M. Antonelli et al., Eur. Phys. J. C 69 (2010) 399 [arXiv:1005.2323 [hep-ph]].

[31] A. Pich, arXiv:1112.4094 [hep-ph].

[32] W. J. Marciano, Phys. Rev. Lett. 93 (2004) 231803 [hep-ph/0402299].

[33] V. Cirigliano and H. Neufeld, Phys. Lett. B 700 (2011) 7 [arXiv:1102.0563 [hep-ph]].

[34] G. Colangelo et al., Eur. Phys. J. C 71 (2011) 1695 [arXiv:1011.4408 [hep-lat]].

[35] I. S. Towner and J. C. Hardy, Rept. Prog. Phys. 73 (2010) 046301.

[36] J. Beringer et al. (Particle Data Group), Phys. Rev. D86 (2012) 010001.

[37] V. Cirigliano, M. Giannotti and H. Neufeld, JHEP 0811 (2008) 006 [arXiv:0807.4507 [hep-ph]].

[38] A. Kastner and H. Neufeld, Eur. Phys. J. C 57 (2008) 541 [arXiv:0805.2222 [hep-ph]].

[39] H. Leutwyler and M. Roos, Z. Phys. C 25 (1984) 91.

[40] M. Jamin, J. A. Oller and A. Pich, JHEP 0402 (2004) 047 [hep-ph/0401080].

[41] V. Cirigliano, G. Ecker, M. Eidemuller, R. Kaiser, A. Pich and J. Portolés, JHEP 0504 (2005) 006 [hep-ph/0503108].

[42] J. Bijnens and P. Talavera, Nucl. Phys. B 669 (2003) 341 [hep-ph/0303103].

[43] P. A. Boyle et al. [RBC-UKQCD Collaboration], Eur. Phys. J. C 69 (2010) 159 [arXiv:1004.0886 [hep-lat]].

[44] V. Cirigliano, G. Ecker and A. Pich, Phys. Lett. B 679 (2009) 445 [arXiv:0907.1451 [hep-ph]].

[45] V. Cirigliano, G. Ecker, H. Neufeld and A. Pich, Eur. Phys. J. C 33 (2004) 369 [hep-ph/0310351]; Phys. Rev. Lett. 91 (2003) 162001 [hep-ph/0307030].

[46] T. Blum et al., Phys. Rev. Lett. 108 (2012) 141601 [arXiv:1111.1699 [hep-lat]]; arXiv:1206.5142 [hep-lat].

[47] T. Blum et al., Phys. Rev. D 84 (2011) 114503 [arXiv:1106.2714 [hep-lat]].

[48] J. R. Batley et al. [NA48 Collaboration], Phys. Lett. B 544 (2002) 97 [hep-ex/0208009], 465 (1999) 335 [hep-ex/9909022]; Eur. Phys. J. C 22 (2001) 231 [hep-ex/0110019].

[49] G. D. Barr et al. [NA31 Collaboration], Phys. Lett. B 317 (1993) 233, 206 (1988) 169.

[50] E. Abouzaid et al. [KTeV Collaboration], Phys. Rev. D 83 (2011) 092001 [arXiv:1011.0127 [hep-ex]], 67 (2003) 012005 [Erratum-ibid. D 70 (2004) 079904] [hep-ex/0208007]; Phys. Rev. Lett. 83 (1999) 22 [hep-ex/9905060]. 
[51] L. K. Gibbons et al., Phys. Rev. Lett. 70 (1993) 1203.

[52] E. Pallante and A. Pich, Phys. Rev. Lett. 84 (2000) 2568 [hep-ph/9911233]; Nucl. Phys. B 592 (2001) 294 [hep-ph/0007208].

[53] E. Pallante, A. Pich and I. Scimemi, Nucl. Phys. B 617 (2001) 441 [hep-ph/0105011].

[54] G. Buchalla, A. J. Buras and M. E. Lautenbacher, Rev. Mod. Phys. 68 (1996) 1125 [hep-ph/9512380].

[55] S. Bertolini, M. Fabbrichesi and J. O. Eeg, Rev. Mod. Phys. 72 (2000) 65 [hep-ph/9802405].

[56] T. Hambye et al., Nucl. Phys. B 564 (2000) 391 [hep-ph/9906434].

[57] A. Pich, hep-ph/0410215.

[58] G. D’Ambrosio and D. Espriu, Phys. Lett. B 175 (1986) 237.

[59] J. L. Goity, Z. Phys. C 34 (1987) 341.

[60] J. Kambor and B. R. Holstein, Phys. Rev. D 49 (1994) 2346 [hep-ph/9310324].

[61] G. Ecker and A. Pich, Nucl. Phys. B 366 (1991) 189.

[62] G. Ecker, A. Pich and E. de Rafael, Phys. Lett. B 189 (1987) 363.

[63] L. Cappiello and G. D’Ambrosio, Nuovo Cim. A 99 (1988) 155.

[64] L. M. Sehgal, Phys. Rev. D 41 (1990) 161.

[65] A. Lai et al. [NA48 Collaboration], Phys. Lett. B 536 (2002) 229 [hep-ex/0205010].

[66] A. G. Cohen, G. Ecker and A. Pich, Phys. Lett. B 304 (1993) 347.

[67] L. Cappiello, G. D’Ambrosio and M. Miragliuolo, Phys. Lett. B 298 (1993) 423.

[68] G. Ecker, A. Pich and E. de Rafael, Phys. Lett. B 237 (1990) 481.

[69] G. Ecker, A. Pich and E. de Rafael, Nucl. Phys. B 303 (1988) 665.

[70] G. D’Ambrosio and J. Portolés, Phys. Lett. B 386 (1996) 403 [hep-ph/9606213]; Nucl. Phys. B 492 (1997) 417 [hep-ph/9610244].

[71] C. Morales (NA48/2), arXiv:0805.3312 [hep-ex].

[72] R.Fantechi, these proceedings.

[73] J. F. Donoghue and F. Gabbiani, Phys. Rev. D 51 (1995) 2187 [hep-ph/9408390].

[74] A. J. Buras, M. E. Lautenbacher, M. Misiak and M. Munz, Nucl. Phys. B 423 (1994) 349 [hep-ph/9402347].

[75] G. Buchalla, G. D’Ambrosio and G. Isidori, Nucl. Phys. B 672 (2003) 387 [hep-ph/0308008].

[76] A. Alavi-Harati et al. [KTeV Collaboration], Phys. Rev. Lett. 93 (2004) 021805 [hep-ex/0309072].

[77] A. J. Buras, M. Gorbahn, U. Haisch and U. Nierste, Phys. Rev. Lett. 95 (2005) 261805 [hep-ph/0508165]; JHEP 0611 (2006) 002 [hep-ph/0603079].

[78] J. Brod, M. Gorbahn and E. Stamou, Phys. Rev. D 83 (2011) 034030 [arXiv:1009.0947 [hep-ph]].

[79] A. V. Artamonov et al. [E949 Collaboration], Phys. Rev. Lett. 101 (2008) 191802 [arXiv:0808.2459 [hep-ex]].

[80] J. K. Ahn et al. [E391a Collaboration], Phys. Rev. D 81 (2010) 072004 [arXiv:0911.4789 [hep-ex]]; Phys. Rev. Lett. 100 (2008) 201802 [arXiv:0712.4164 [hep-ex]].

[81] M. Fiorini, J. Comfort and A. Norman, these proceedings. 